\title{
An unexpected ring formation in morphine chemistry
}

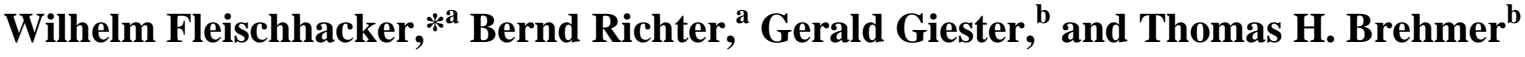 \\ ${ }^{a}$ Institute of Pharmaceutical Chemistry, University of Vienna, Althanstraße 14, A-1090 Wien, \\ Austria \\ E-mail: wilhelm.fleischhacker@univie.ac.at \\ ${ }^{b}$ Institute of Crystallography and Mineralogy, University of Vienna, Althanstraße 14, A-1090 \\ Wien, Austria \\ E-mail: gerald.giester@univie.ac.at
}

Dedicated to Prof. Fritz Sauter on the occasion of his $70^{\text {th }}$ birthday

(received 23 Dec 00; accepted 29 Oct 01; published on the web 06 Nov 01)

\begin{abstract}
Reaction of (7R)- $N$-benzyl- $N$-nor-7-bromoneopinone dimethyl acetal (2) with primary or secondary amines (RNH2, R1R2NH) resulted in 9-amino-substituted hasubanan derivatives; in particular, modification of the 9-(2-hydroxyethyl)amino derivative in subsequent steps gave products 8 with a 9-(oxazolidin-2'-one-3'-yl) substituent. ${ }^{1} \mathrm{H}$ and ${ }^{13} \mathrm{C}$ NMR spectra did not provide definitive evidence for the supposed structure and reaction mechanism, but X-ray analyses of compounds $\mathbf{8 a}$ and $\mathbf{8 d}$ confirmed both structures.
\end{abstract}

Keywords: Morphine derivatives, neopine derivatives, hasubanan, ring formation, 9(oxazolidin-2'-one-3'-yl)-substitution, X-ray structure analysis

\section{Introduction}

It is known that the position of basic nitrogen in the morphine scaffold as well as the type of its substituents are of great importance with regard to the interaction with the anionic site of the receptor. The goal of our approach was to introduce a second basic group in the vicinity to the nitrogen attached to C-9. The subsequent step should turn the original nitrogen atom into a nonbasic group, incapable of interaction with the anionic site of the receptor. In this way the scaffold nitrogen would be changed to inefficiency whereas the newly inserted one could be capable of pharmacologic interactions.

Two more points of consideration should be mentioned: 1. A second nitrogen offers the advantage to insert an intramolecular bridge. 2 . The synthesis of dimers by inserting suitable bivalent reagents should be possible; such compounds are known to possess higher 
antinociceptive potency and receptor selectivity by entropic reasons. ${ }^{1}$

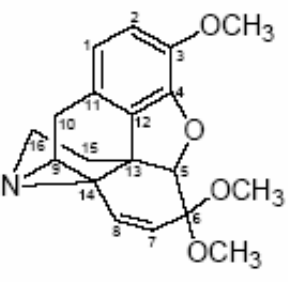

1

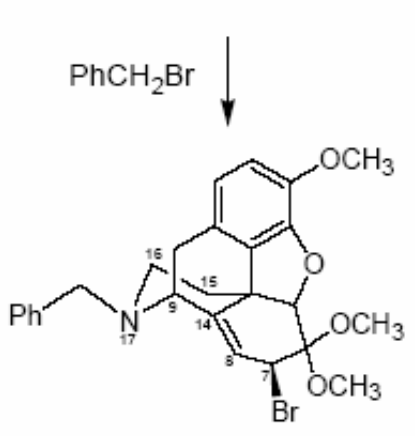

2

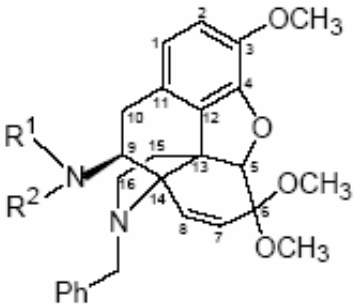

$3 a, b ; 3 c \rightarrow 3 d \longrightarrow[3 e]$
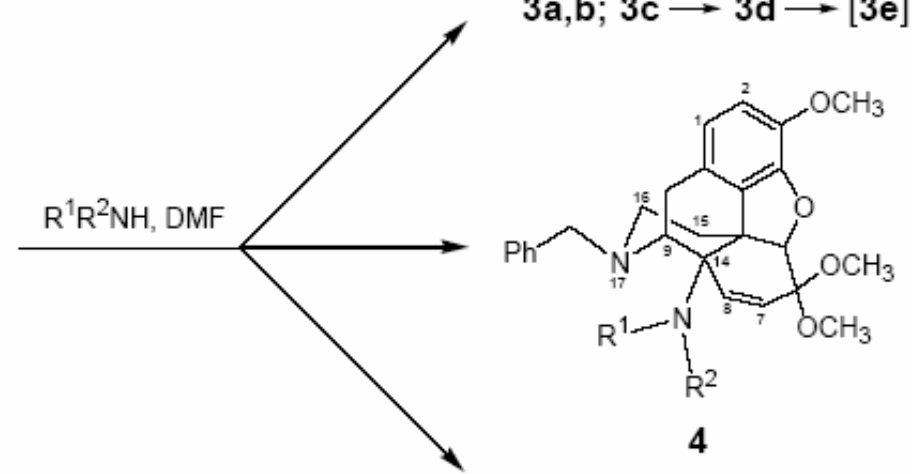

4

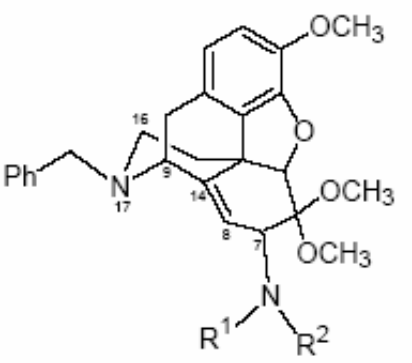

5

\section{Scheme 1}

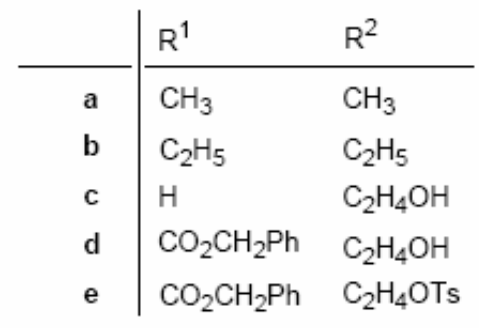

We started as follows (Scheme 1): Reaction of the known 14,17-cyclonorcodeinone dimethyl acetal $\mathbf{1}^{2}$ with benzyl bromide yielded quantitatively the neopinone derivative $2 .{ }^{3}$ Generally, hydrolysis, methanolysis, acetolysis, i.e. nucleophilic substitution of 7-bromoneopinone or 14bromocodeinone acetal derivatives are assumed to occur via an ionic intermediate (a quaternary aziridinium ion derived from 1), which results in a mixture of 7-, 14- and 9-substituted derivatives. ${ }^{4}$ Therefore, the straightforward reaction with primary and secondary amines furnishing single, defined products was a surprise at first sight. Inspection of the NMR spectra of the product eliminated structure 5 , but it was not possible to discriminate unambiguously between amine substitution at C-9 (as in 3) or C-14 (as in 4) by any routine analytical method. Only the ${ }^{1} \mathrm{H}$ NMR spectrum of derivative 3d, obtained later, gave evidence in support of the proposed structure based on the paramagnetic shift of the H-9 signal as a consequence of carbamate formation. We expected that synthetic steps to be carried out at a later stage and aiming at cyclisation by bridging both nitrogen atoms would support this indication, but at last the X-ray analyses of $\mathbf{8 a}$ and $\mathbf{8 d}$ confirmed the substitution at C-9. 


\section{Results and Discussion}

In regard to our experience with codeinone derivatives, bridge building between both nitrogen atoms at C-9 and C-14 should be possible. ${ }^{5}$ In $3 c$ and $4 c\left(\mathrm{R}_{1}=\mathrm{H}, \mathrm{R}_{2}=\mathrm{CH}_{2} \mathrm{CH}_{2} \mathrm{OH}\right)$ the hydroxyl group is expected to become a good nucleofuge upon conversion into the tosylate such as $3 \mathbf{e}$ or 4e, thus facilitating ring closure with the other nitrogen ( $\mathrm{N}-17$ of the original morphine scaffold) to form ammonium salt $\mathbf{6}$ or $\mathbf{7}$, respectively (Scheme 2), which in turn, could afford a dibasic compound after detachment of the benzyl groups in a subsequent step.

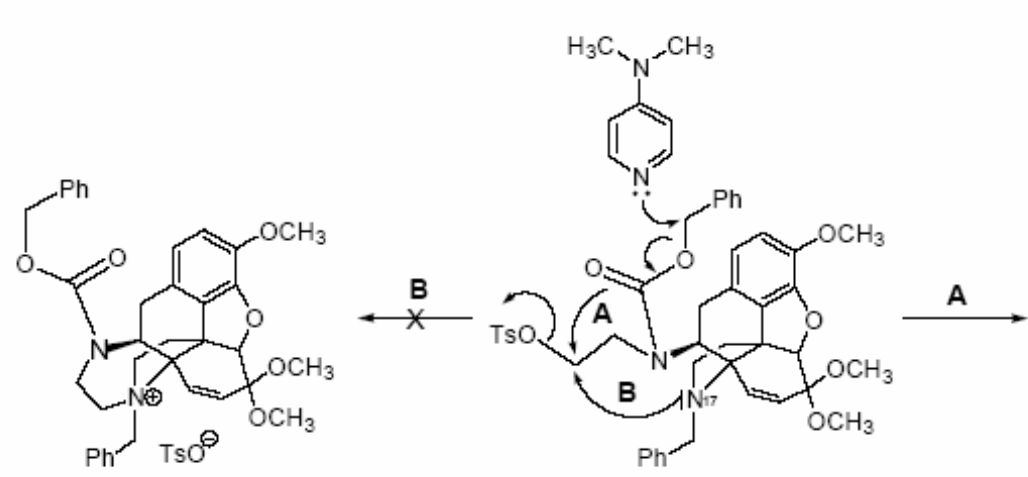

6

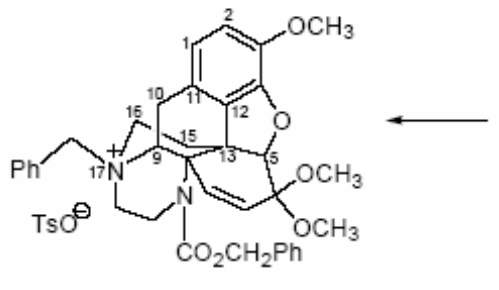

7
$[3 e]$

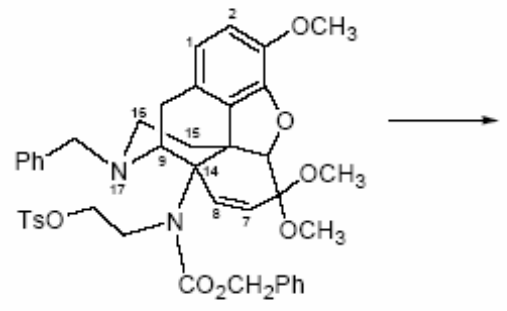

[4e]

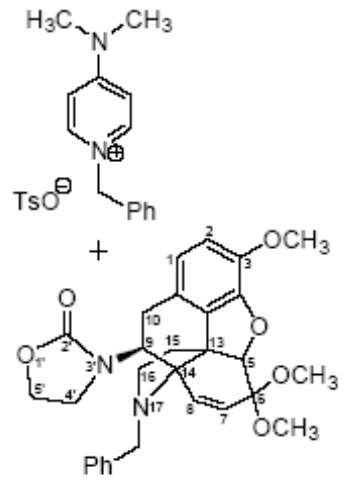

$8 \mathrm{a}$

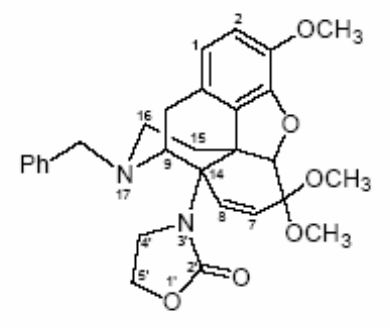

9

\section{Scheme 2}

At this stage we engaged in some mechanistic considerations to evaluate the possibility of an alternative ring formation from $3 \mathbf{e}$ or $\mathbf{4 e}$ (bond lengths, steric energies). It must be argued that at first sight there seemed to be no energy preference for ring formation taking place from a newly inserted $N$-substituent at C-9 to the rearranged $\mathrm{N}-17$, now located at C-14 (in 3), or alternatively from the alkylamino substituent at $\mathrm{C}-14$ to $\mathrm{N}-17$ bonded to $\mathrm{C}-9$ as in the original scaffold (in 4). In fact, computation (MM2 force field) of the energies of the alternative bases derived from structures 6 and 7 gave almost identical results (14.7 vs. $14.9 \mathrm{kJmol}_{-1}$ ), whereas the quaternary ammonium salts 6 and 7 revealed a rather low steric energy of $16.4 \mathrm{kJmol}^{-1}$ for $\mathbf{6}$, vs. $18.6 \mathrm{kJmol}^{-1}$ for 7 , obviously due to the more crowded topology caused by substitution with the bulkier Zgroup in the vicinity of the quaternary $\mathrm{C}-14$. Since the reaction did not work efficiently with the unprotected secondary amine 3c or 4c, the benzyloxycarbonyl group (Z-group) was introduced as $\mathrm{R}_{1}$ to give $\mathbf{3 d}$ or $\mathbf{4 d}$. The subsequent tosylation step to form $3 \mathbf{e}$ or $\mathbf{4 e}$ was carried out without 
isolation of the resulting intermediate and was expected to facilitate the desired intramolecular reaction by increasing the electrophilicity of the terminal methylene group (Scheme 2).

Surprisingly, we did not obtain the quaternary ammonium salt 6 or 7, but rather a product in high yield and with concomitant loss of one benzyl group. Later this product was identified as the oxazolidinone derivative $\mathbf{8 a}$ (vide infra). At first, we were not aware of this and believed that a diazine moiety had been formed, and keeping in mind the loss of one benzyl group we set out to distinguish between a product structure derived from either $\mathbf{6}$ or $\mathbf{7}$.

It is noteworthy to mention that the decisive condition for this unique reaction leading to 8a is the presence of dimethylaminopyridine (DMAP) in the tosylation step which triggered the entire reaction sequence: nucleophilic attack of DMAP at the $O$-benzyl methylene group induced $\mathrm{CH}_{2}-\mathrm{O}-$ bond cleavage and ring closure by the carboxyl oxygen at the tosyloxymethylene group with displacement of the tosylate anion. The benzyl group of the Z-group was consumed by DMAP affording the $N$-benzyl-4-(dimethyl-amino)pyridinium ion, which was precipitated as the perchlorate salt and analyzed after crystallisation. Without the assistance of DMAP no reaction was detectable.

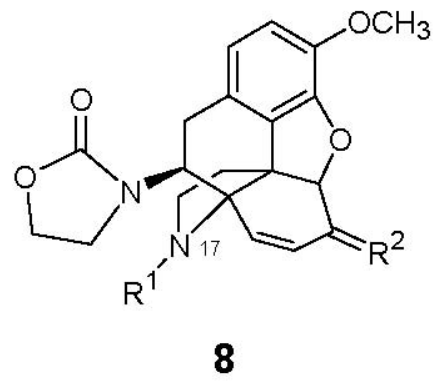

\begin{tabular}{l|ll}
$\mathbf{8}$ & $\mathrm{R}^{1}$ & $\mathrm{R}^{2}$ \\
\cline { 2 - 3 } $\mathbf{a}$ & $\mathrm{CH}_{2} \mathrm{Ph}$ & $\left(\mathrm{OCH}_{3}\right)_{2}$ \\
b & $\mathrm{H}$ & $\left(\mathrm{OCH}_{3}\right)_{2}$ \\
c & $\mathrm{CH}_{3}$ & $\left(\mathrm{OCH}_{3}\right)_{2}$ \\
d & $\mathrm{CH}_{2} \mathrm{CH}=\mathrm{CH}_{2}$ & $\left(\mathrm{OCH}_{3}\right)_{2}$ \\
e & $\mathrm{CH}_{2} \mathrm{Ph}$ & $\mathrm{O}$ \\
f & $\mathrm{H}$ & $\mathrm{O}$ \\
g & $\mathrm{CH}_{3}$ & $\mathrm{O}$ \\
h & $\mathrm{CH}_{2} \mathrm{CH}=\mathrm{CH}_{2}$ & $\mathrm{O}$ \\
i & $\mathrm{CO}_{2} \mathrm{CH}_{2} \mathrm{Ph}$ & $\left(\mathrm{OCH}_{3}\right)_{2}$
\end{tabular}

Compound $\mathbf{8 a}$ was debenzylated to $\mathbf{8 b}$ by hydrogenation over $\mathrm{Pd} / \mathrm{C}$-catalyst in acetic acid. Finally, the structure elucidation of $\mathbf{8 d}$ and $\mathbf{8 a}$ by X-ray analyses ensured that the oxazolidine derivative had in fact been formed. This result eliminates structure $\mathbf{9}$ and that of its conceivable precursor $4 \mathbf{e}$.

\section{Crystal structure discussion}

Eventually, the structure of compounds 8a and 8d was proven by X-ray analysis (Figures 1 and 2). 


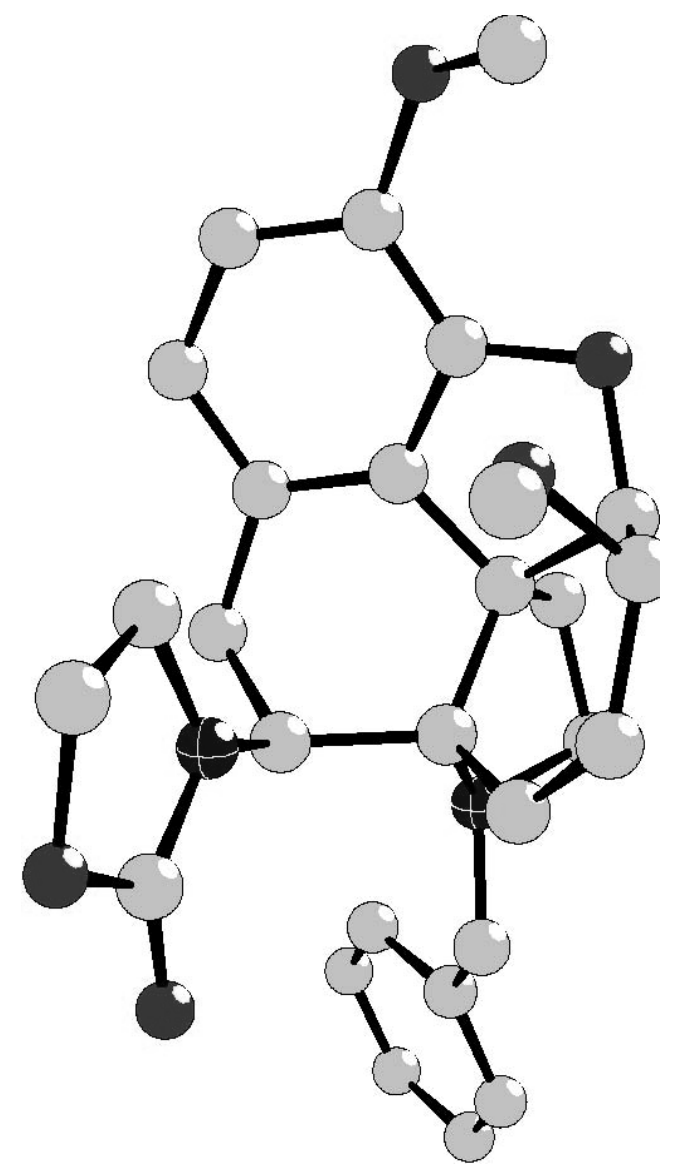

Figure 1. Perspective view of compound 8a.

The crystal data are summarized in the Experimental part. Lists of structure factors, atomic coordinates and thermal components for non-hydrogen atoms and hydrogen atom parameters are available from T.H.B. and have been deposited at the Cambridge Crystallograpgic Data Centre. All bond lengths and angles in the molecules 8a and 8d lie within the expected ranges. The phenyl rings $(\mathrm{C} 1, \mathrm{C} 2, \mathrm{C} 3, \mathrm{C} 4, \mathrm{C} 11, \mathrm{C} 12)$ and $(\mathrm{C} 24-\mathrm{C} 29)$ are planar, whereas the other sixmembered rings are characterized by classical conformations (chair, boat, twist-boat) with deviations of some ring atoms. The five-membered rings $(\mathrm{O} 2, \mathrm{C} 5, \mathrm{C} 13, \mathrm{C} 12, \mathrm{C} 4)$ and $(\mathrm{C} 13, \mathrm{C} 14, \mathrm{~N} 1, \mathrm{C} 16, \mathrm{C} 15)$ have envelope conformations with $\mathrm{C} 5$ and $\mathrm{C} 16$, respectively, deviating from the planes defined by the other ring atoms. The oxazolidine ring (N2,C17,O4,C18,C19) adopts an envelope conformation only in compound 8a, where C18 deviates from the ring plane. In structure 8d this ring is planar. 


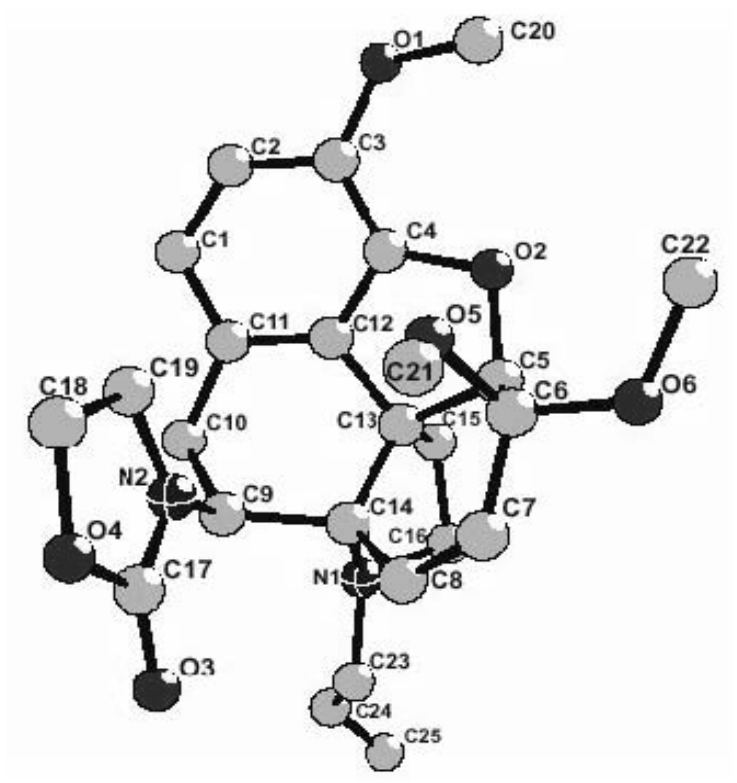

Figure 2 Perspective view of compound 8d with atom labeling scheme.

\section{Experimental Section}

General Procedures. Melting points: Kofler hot stage microscope; column chromatography was performed on silica gel (Kieselgel 60, 70-230 mesh; Merck). IR spectra were recorded on a Perkin Elmer Spectrum 1000 instrument; NMR spectra were recorded on a Varian Unity Plus 300 spectrometer; mass spectra were measured on a Shimadzu QP-5000 mass spectrometer (EI, $70 \mathrm{eV})$. Elemental analyses were performed by the Laboratory of Microanalysis, Institute for Physical Chemistry of the University of Vienna. Energy computations were accomplished using the CS Chem 3D Pro ${ }^{\circledR}$ program (MM2 force field), Cambridge, MA.

(7R)- $N$-Benzyl- $N$-nor-7-bromoneopinone dimethyl acetal (2). Benzyl bromide $(0.75 \mathrm{~mL}$, $6.3 \mathrm{mmol})$ was added to 14,17-cyclonorcodeinone dimethyl acetal $(1,2.0 \mathrm{~g}, 6.11 \mathrm{mmol})$ in $\mathrm{CH}_{2} \mathrm{Cl}_{2}(20 \mathrm{~mL})$ and stirred at $25{ }^{\circ} \mathrm{C}$ for $6 \mathrm{~h}$. After addition of a saturated aqueous solution of $\mathrm{NaHCO}_{3}(5 \mathrm{~mL})$ stirring was continued for $1 \mathrm{~h}$. The $\mathrm{CH}_{2} \mathrm{Cl}_{2}$ layer was separated, washed with water, dried over $\mathrm{Na}_{2} \mathrm{SO}_{4}$, and concentrated. The residual pale oil 2 (3.0 g, 98\%) was purified over silica gel (petrol ether/ethyl acetate/triethylamine 10:2:1, $\left.\mathrm{R}_{f}=0.43\right) .{ }^{1} \mathrm{H} \mathrm{NMR}\left(\mathrm{CDCl}_{3}\right): \delta$ 7.45-7.20 (m, 5H, $\left.\mathrm{C}_{6} \mathrm{H}_{5}\right), 6.80\left(\mathrm{~d}, J_{1,2}=8.1 \mathrm{~Hz}, 1 \mathrm{H}, \mathrm{H}-2\right), 6.55\left(\mathrm{~d}, J_{1,2}=8.1 \mathrm{~Hz}, 1 \mathrm{H}, \mathrm{H}-1\right), 5.77$ $\left(\mathrm{d}, J_{7,8}=6.3 \mathrm{~Hz}, 1 \mathrm{H}, \mathrm{H}-8\right), 5.22(\mathrm{~s}, 1 \mathrm{H}, \mathrm{H}-5), 4.59$ (d, J7,8 $\left.=6.3 \mathrm{~Hz}, 1 \mathrm{H}, \mathrm{H}-7\right), 3.90$ (s, 3H, 3$\left.\mathrm{OCH}_{3}\right), 4.01,3.92\left(\mathrm{AB}, J_{\text {gem }}=13.4 \mathrm{~Hz}, 2 \mathrm{H}\right.$, benzyl-HАНв $), 3.54\left(\mathrm{~d}, J_{9,10 \beta}=7.1 \mathrm{~Hz}, 1 \mathrm{H}, \mathrm{H}-9\right)$, $3.53\left(\mathrm{~s}, 3 \mathrm{H}, 6 \alpha-\right.$ or $\left.6 \beta-\mathrm{OCH}_{3}\right), 3.21\left(\mathrm{~d}, J_{\mathrm{gem}}=18.2 \mathrm{~Hz}, 1 \mathrm{H}, \mathrm{H}_{\alpha}-10\right), 3.03\left(\mathrm{~m}, 1 \mathrm{H}, \mathrm{H}_{\mathrm{A}}-16\right), 3.02(\mathrm{~s}$, $3 \mathrm{H}, 6_{\alpha-}$ or $\left.6_{\beta}-\mathrm{OCH}_{3}\right), 2.86\left(\mathrm{dd}, J_{9,10 \beta}=7.1 \mathrm{~Hz}, J_{\text {gem }}=18.2 \mathrm{~Hz}, 1 \mathrm{H}, \mathrm{H}_{\beta}-10\right), 2.68(\mathrm{~m}, 1 \mathrm{H}, \mathrm{Hв}-16)$, $2.50(\mathrm{~m}, 1 \mathrm{H}, \mathrm{HA}-15), 1.69(\mathrm{~m}, 1 \mathrm{H}, \mathrm{HB}-15) ;{ }^{13} \mathrm{C} \mathrm{NMR}\left(\mathrm{CDCl}_{3}\right): \delta 145.68(\mathrm{C}-4), 143.80(\mathrm{C}-14)$, 142.01 (C-3), 138.91 (CPh-1), 131.25 (C-12), 128.68, 128.20 (CPh-2,6, CPh-3,5), 127.04 (C-11), 126.89 (CPh-4), 119.04 (C-1), 117.00 (C-8), 114.60 (C-2), 98.48 (C-6), 91.89 (C-5), 57.98 (C-9), 
57.02 (benzyl- $\left.\mathrm{CH}_{2}\right), 57.02\left(3-\mathrm{OCH}_{3}\right), 50.42,49.50\left(6 \alpha-\right.$ and $\left.6 \beta-\mathrm{OCH}_{3}\right), 46.69(\mathrm{C}-13), 46.61(\mathrm{C}-$ 7), 43.46 (C-16), 34.79 (C-15), 31.86 (C-10); MS: m/z 497, 499 (1:1). Anal Calcd for $\mathrm{C}_{26} \mathrm{H}_{28} \mathrm{BrNO}_{4}$ (498.42): C, 62.66; H, 5.66; Br, 16.03; N, 2.81. Found: C, 62.39; H, 5.65; Br, 15.95; N, 2.81.

(9S)-17-Benzyl-4,5-epoxy-9-diethylamino-3-methoxyhasubanan-6-one dimethyl acetal (3b). To a solution of $2(580 \mathrm{mg}, 1.16 \mathrm{mmol})$ in DMF $(15 \mathrm{~mL})$ was added dry diethylamine $(0.6 \mathrm{~mL}$, excess), and the mixture was stirred under Ar at $120{ }^{\circ} \mathrm{C}$ for $4 \mathrm{~h}$. After evaporation and flash chromatography (petroleum ether/ethyl acetate/triethylamine 12:2:0.5) 3b (150 mg, 26\%) was obtained as an analytically pure oil. Another pure fraction was 3a (120 mg, 22\%; formed from dimethylamine present in purchased and unpurified diethylamine; Merck 803010, "z. Synthese"). ${ }^{1} \mathrm{H}$ NMR $\left(\mathrm{CDCl}_{3}\right): \delta 7.38\left(\mathrm{~m}, 5 \mathrm{H}, \mathrm{C}_{6} \mathrm{H}_{5}\right), 6.73\left(\mathrm{~d}, J_{1,2}=8.1 \mathrm{~Hz}, 1 \mathrm{H}, \mathrm{H}-2\right), 6.67\left(\mathrm{~d}, J_{1,2}=8.1 \mathrm{~Hz}\right.$, $1 \mathrm{H}, \mathrm{H}-1), 6.26$ (d, J7,8 = 11.1 Hz, 1H, H-8), 5.88 (d, J7,8=11.1 Hz, 1H, H-7), 4.64 (s, 1H, H-5), $4.32\left(\mathrm{~d}, J_{\text {gem }}=13.5 \mathrm{~Hz}, 1 \mathrm{H}\right.$, benzyl-HA $), 3.98\left(\mathrm{~s}, 3 \mathrm{H}, 3-\mathrm{OCH}_{3}\right), 3.58(\mathrm{~m}, 1 \mathrm{H}, \mathrm{H}-9), 3.56(\mathrm{~s}, 3 \mathrm{H}$, $6_{\alpha}$ - or $\left.6_{\beta}-\mathrm{OCH}_{3}\right), 3.49\left(\mathrm{~d}, J_{\mathrm{gem}}=13.5 \mathrm{~Hz}, 1 \mathrm{H}\right.$, benzyl-Hв), $3.11\left(\mathrm{~s}, 3 \mathrm{H}, 6 \alpha_{-}\right.$or $\left.6 \beta-\mathrm{OCH}_{3}\right), 2.99$ (d, $\left.J_{\text {gem }}=15.6 \mathrm{~Hz}, 1 \mathrm{H}, \mathrm{H}_{\alpha}-10\right), 2.87\left(\mathrm{dd}, J_{9,10 \beta}=4.95 \mathrm{~Hz}, J_{\text {gem }}=15.6 \mathrm{~Hz}, 1 \mathrm{H}, \mathrm{H}_{\beta}-10\right), 2.64(\mathrm{~m}, 1 \mathrm{H}$, HA-16), 2.27 (m, 1H, Нв-16), 2.11 (broad m, 4H, 2 x $\mathrm{CH}_{2} \mathrm{CH}_{3}$ ), 2.02 (m, 1H, HA-15), 1.62 (m, 1H, Hв-15), 0.91 (t, 6H, $\left.2 \times \mathrm{CH}_{2} \mathrm{CH}_{3}\right) ;{ }^{13} \mathrm{C} \mathrm{NMR}\left(\mathrm{CDCl}_{3}\right): \delta 146.21$ (C-4), 141.48 (C-3), 140.63 (CPh-1), 133.74 (C-8), 132.19 (C-12), 128.26 (CPh-2,6), 128.08 (CPh-3,5), 127.44 (C-11), 126.82 (CPh-4), 125.29 (C-7), 118.79 (C-1), 115.23 (C-2), 94.49 (C-6), 94.36 (C-5), 67.06 (C-9), 65.97 (C-14), 57.47 (3-OCH3), 53.23 (benzyl-CH2), 52.39 (C-13), 50.08, $48.78\left(6 \alpha^{-}, 6 \beta-\mathrm{OCH}_{3}\right), 46.55$ (C-16), $44.29\left(\mathrm{CH}_{2} \mathrm{CH} 3\right), 40.75(\mathrm{C}-15), 23.64(\mathrm{C}-10), 15.00 \quad\left(\mathrm{CH}_{2} \mathrm{CH}_{3}\right) ; \mathrm{MS}: \mathrm{m} / \mathrm{z} \quad 490$, $\mathrm{C}_{30} \mathrm{H}_{38} \mathrm{~N}_{2} \mathrm{O}_{4}$ (490.63).

(9S)-17-benzyl-4,5-epoxy-3-methoxy-9-dimethylaminohasubanan-6-one dimethyl acetal (3a). ${ }^{1} \mathrm{H}$ $\operatorname{NMR}\left(\mathrm{CDCl}_{3}\right): \delta 7.37\left(\mathrm{~m}, 5 \mathrm{H}, \mathrm{C}_{6} \mathrm{H}_{5}\right), 6.72\left(\mathrm{~d}, J_{1,2}=8.0 \mathrm{~Hz}, 1 \mathrm{H}, \mathrm{H}-2\right), 6.69$ (d, J1,2 = 8.0 Hz, 1H, H-1), 6.21 (d, $\left.J_{7,8}=11.1 \mathrm{~Hz}, 1 \mathrm{H}, \mathrm{H}-7\right), 5.93$ (d, J7,8 $\left.=11.1 \mathrm{~Hz}, 1 \mathrm{H}, \mathrm{H}-8\right), 4.65$ (s, 1H, H-5), 4.28 (d, Jgem $=13.5 \mathrm{~Hz}, 1 \mathrm{H}$, benzyl-HA), $3.97\left(\mathrm{~s}, 3 \mathrm{H}, 3-\mathrm{OCH}_{3}\right), 3.56\left(\mathrm{~s}, 3 \mathrm{H}, 6 \alpha-\right.$ or $\left.6 \beta-\mathrm{OCH}_{3}\right), 3.50\left(\mathrm{~d}, J_{\mathrm{gem}}=13.5 \mathrm{~Hz}, 1 \mathrm{H}\right.$, benzylНв), $3.24(\mathrm{~m}, 1 \mathrm{H}, \mathrm{H}-9), 3.09$ (s, 3H, $6 \alpha-$ or $\left.6 \beta-\mathrm{OCH}_{3}\right), 2.97\left(\mathrm{~m}, J_{\text {gem }}=15.6 \mathrm{~Hz}, 2 \mathrm{H}, \mathrm{H}-10\right), 2.68$ (m, $1 \mathrm{H}, \mathrm{H}_{\alpha}-16$ or $\left.\mathrm{H}_{\beta}-16\right), 2.31$ (m, $1 \mathrm{H}, \mathrm{H}_{\alpha}-16$ or $\left.\mathrm{H}_{\beta}-16\right), 2.05$ (m, $1 \mathrm{H}_{1} \mathrm{H}_{\alpha}-15$ or $\left.\mathrm{H}_{\beta}-15\right), 1.97$ (s, $\left.6 \mathrm{H}, 2 \times \mathrm{CH}_{3}\right), 1.62\left(\mathrm{~m}, 1 \mathrm{H}, \mathrm{H}-15 \alpha\right.$ or $\left.\mathrm{H}-15_{\beta}\right) ;{ }^{13} \mathrm{C} \mathrm{NMR}\left(\mathrm{CDCl}_{3}\right): \delta 146.20(\mathrm{C}-4), 141.46(\mathrm{C}-3)$, 140.47 (Cph-1), 133.31 (C-8), 132.19 (C-12), 128.29 (CPh-2,6), 127.98 (Cph-3,5), 127.14 (C-11), 126.85 (CPh-4), 126.76 (C-7), 119.05 (C-1), 115.25 (C-2), 94.72 (C-6), 94.62 (C-5), 69.90 (C-9), $65.88(\mathrm{C}-14), 57.44\left(3-\mathrm{OCH}_{3}\right), 52.82\left(\right.$ benzyl- $\left.\mathrm{CH}_{2}\right), 52.16(\mathrm{C}-13), 50.19,48.27\left(6 \alpha-, 6 \beta-\mathrm{OCH}_{3}\right)$, 46.69 (C-16), $43.12\left(\mathrm{CH}_{3}\right), 41.49$ (C-15), 21.61 (C-10); MS: $m / z$ 462, $\mathrm{C}_{30} \mathrm{H}_{34} \mathrm{~N}_{2} \mathrm{O}_{4}(462.59)$.

(9S)-17-Benzyl-4,5-epoxy-9-hydroxyethylamino-3-methoxyhasubanan-6-one dimethyl acetal (3c). A solution of $2(6.08 \mathrm{~g}, 12.21 \mathrm{mmol})$ and aminoethanol $(7.4 \mathrm{~mL}$, excess) in dry DMF $(100 \mathrm{~mL})$ was stirred under Ar at $130{ }^{\circ} \mathrm{C}$ (bath temperature) for $4 \mathrm{~h}$. After evaporation of the solvent the residue was taken up with ethyl acetate. The organic extract was washed with brine and water, dried with $\mathrm{Na}_{2} \mathrm{SO}_{4}$, and after removal of the solvent under reduced pressure 3c was obtained a pale orange oil (5.73 g, 98\%), which was sufficiently pure for further reactions. An analytical sample was crystallized from ethanol and gave colourless crystals, $\mathrm{mp}$ 
158-160 ${ }^{\circ} \mathrm{C} ;{ }^{1} \mathrm{H}$ NMR $\left(\mathrm{CDCl}_{3}\right): \delta 7.37-7.27\left(\mathrm{~m}, 5 \mathrm{H}, \mathrm{C}_{6} \mathrm{H}_{5}\right), 6.74\left(\mathrm{~d}, \mathrm{~J}_{1,2}=8.1 \mathrm{~Hz}, 1 \mathrm{H}, \mathrm{H}-1\right), 6.73$ $\left(\mathrm{d}, J_{1,2}=8.1 \mathrm{~Hz}, 1 \mathrm{H}, \mathrm{H}-2\right), 6.19$ (d, $\left.J_{7,8}=10.8 \mathrm{~Hz}, 1 \mathrm{H}, \mathrm{H}-7\right), 6.00$ (d, $\left.J_{7,8}=10.8 \mathrm{~Hz}, 1 \mathrm{H}, \mathrm{H}-8\right)$, $4.62(\mathrm{~s}, 1 \mathrm{H}, \mathrm{H}-5), 4.27\left(\mathrm{~d}, J_{\mathrm{gem}}=13.5 \mathrm{~Hz}, 1 \mathrm{H}\right.$, benzyl-HA), $3.95\left(\mathrm{~s}, 3 \mathrm{H}, 3-\mathrm{OCH}_{3}\right), 3.59(\mathrm{~s}, 3 \mathrm{H}, 6 \alpha-$ or $\left.6 \beta-\mathrm{OCH}_{3}\right), 3.52\left(\mathrm{~d}, 1 \mathrm{H}\right.$, benzyl-Hв), 3.45, $3.25\left(\mathrm{~m}, \mathrm{~m}, 1 \mathrm{H}, 1 \mathrm{H}, \mathrm{OCH}_{\mathrm{AH}}\right), 3.21\left(\mathrm{~d}, J_{\mathrm{gem}}=\right.$ $\left.14.7 \mathrm{~Hz}, 1 \mathrm{H}, \mathrm{H}_{\alpha}-10\right), 3.19$ (broad s, $\left.1 \mathrm{H}, \mathrm{H}-9\right), 3.04\left(\mathrm{~s}, 3 \mathrm{H}, 6 \alpha-\right.$ or $\left.6{ }_{\beta}-\mathrm{OCH}_{3}\right), 2.88$ (broad d, $1 \mathrm{H}$, $\left.\mathrm{H}_{\beta}-10\right), 2.75$ (m, 1H, Hax-16), 2.66, 2.48 (m, m, 1H, 1H, NCHАНв), 2.34 (m, 1H, Heq-16), 2.12 $(\mathrm{m}, 1 \mathrm{H}, \mathrm{Hax}-15), 1.60(\mathrm{~m}, 1 \mathrm{H}, \mathrm{Heq}-15) .{ }^{13} \mathrm{C} \mathrm{NMR}\left(\mathrm{CDCl}_{3}\right): \delta 146.12$ (C-4), $141.78(\mathrm{C}-3), 140.03$ (CPh-1), 133.16 (C-12), 132.38, 130.58 (C-7, C-8), 128.23, 127.28 (CPh-2,6, CPh-3,5), 126.83 (CPh-4), 123.64 (C-11), 120.53 (C-1), 114.42 (C-2), 95.46 (C-6), 94.63 (C-5), 68.44 (C-14), $60.16\left(\mathrm{OCH}_{2}\right), 59.63(\mathrm{C}-9), 57.17\left(3-\mathrm{OCH}_{3}\right), 53.09$ (benzyl-CH$), 52.27(\mathrm{C}-13), 50.82,48.49$ $\left(6 \alpha-\right.$ and $\left.{ }_{6} \beta-\mathrm{OCH}_{3}\right), 47.91(\mathrm{C}-16), 47.43\left(\mathrm{NCH}_{2}\right), 41.86(\mathrm{C}-15), 27.45(\mathrm{C}-10)$; MS: $\mathrm{m} / \mathrm{z}(\%) 478$ (0.35), 446 (100; $\left.\mathrm{M}-\mathrm{CH}_{3} \mathrm{OH}\right)$. Anal. Calcd. for $\mathrm{C}_{28} \mathrm{H}_{34} \mathrm{~N}_{2} \mathrm{O}_{5}$ (478.59): C, 70.27; H, 7.16; N, 5.85. Found: C, 70.26; H, 7.30; N, 5.78.

\section{(9S)-17-Benzyl-( $N$-benzyloxycarbonyl)-4,5-epoxy-9-hydroxyethylamino-3-meth-}

oxyhasubanan-6-one dimethyl acetal (3d). To a solution of 3c $(3.10 \mathrm{~g}, 6.5 \mathrm{mmol})$ in acetone $(65 \mathrm{~mL})$ were added $\mathrm{K}_{2} \mathrm{CO}_{3}(1.0 \mathrm{~g}, 7.2 \mathrm{mmol})$ and benzyl chloroformate $(1 \mathrm{~mL}, 1.2 \mathrm{~g}, 7.0 \mathrm{mmol})$, and the mixture was stirred at $40{ }^{\circ} \mathrm{C}$ (bath temperature) under Ar for $2 \mathrm{~h}$. After concentration, the crude residue $(3.81 \mathrm{~g}, 96 \%$ ) was found to be sufficiently pure for further reactions. For analytical purpose a sample was purified by flash chromatography on silica gel (petrol ether/ethyl acetate/triethylamine 5:2:1). The NMR spectra show two sets of spectra due to the presence of amide rotamers. ${ }^{1} \mathrm{H}$ NMR $\left(\mathrm{CDCl}_{3}\right): \delta 7.33-7.08\left(\mathrm{~m}, 10 \mathrm{H}, 2 \mathrm{C}_{6} \mathrm{H}_{5}\right), 6.76-6.63(\mathrm{AB}, 2 \mathrm{H}, \mathrm{H}-1, \mathrm{H}-$ 2), 5.93-5.71 (m, 2H, H-7, H-8), 5.26-5.04 (m, 2H, benzyl-CH CH $^{2}$ 4.99, 4.82 (d, d, 1H, 1H, H-9), 4.60, $4.56(\mathrm{~s}, \mathrm{~s}, 1 \mathrm{H}, 1 \mathrm{H}, \mathrm{H}-5), 4.19\left(\mathrm{~d}, 1 \mathrm{H}, J_{\mathrm{gem}}=13.7 \mathrm{~Hz}\right.$, benzyl-HA), $3.92\left(\mathrm{~s}, 3 \mathrm{H}, 3-\mathrm{OCH}_{3}\right)$, $3.50\left(\mathrm{~s}, 3 \mathrm{H}, 6 \alpha-\right.$ or $\left.6 \beta-\mathrm{OCH}_{3}\right), 3.46-3.12\left(\mathrm{~m}, 5 \mathrm{H}, \mathrm{H}_{\mathrm{A}}-10, \mathrm{CH}_{2} \mathrm{CH}_{2} \mathrm{O}\right), 3.05\left(\mathrm{~d}, 1 \mathrm{H}, J_{\mathrm{gem}}=13.7\right.$ $\mathrm{Hz}$, benzyl-Нв), 3.02, 2.96 (s, s, 3H, 3H, $26_{\alpha}$ - and $\left.6 \beta_{\beta}-\mathrm{OCH}_{3}\right), 2.85-2.52$ (m, 1H, Нв-10, НА-16), 2.30-1.79 (m, 1H, H-15А, Нв-16), 1.65-1.45 (m, 1H, H-15в). ${ }^{13} \mathrm{C} \mathrm{NMR}\left(\mathrm{CDCl}_{3}\right): \delta 158.49$, 158.00 (2 CO), 146.49, 126.41 (2 C-4), 142.26 (C-3), 140.39, 139.90 (2 N-benzyl-Cph-1), 136.46, 135.11 (2 O-benzyl-CPh-1), 132.92, 132.12 (2 C-12), 130.75, 130.48, 130.14 (C-7, C-8), 128.63, 128.48, 128.41, 128.17, 128.09, 127.90, 127.77 (CPh), 126.67 (2 CPh-4), 124.45, 123.86 (2 C-11), 119.02, 118.49 (2 C-1), 114.87, 114.65 (2 C-2), 94.43, 94.15 (2 C-5), 94.27 (C-6), 67.85 (C-14), 68.57, 67.26 (2 benzyl- $\left.\mathrm{CH}_{2} \mathrm{O}\right), 64.23,62.03\left(2 \mathrm{CH}_{2} \mathrm{O}\right), 60.53,60.12$ (2 C-9), 57.24 $\left(3-\mathrm{OCH}_{3}\right), 52.40,52.03\left(\mathrm{CH}_{2} \mathrm{~N}\right.$, benzyl- $\left.\mathrm{CH}_{2} \mathrm{~N}\right), 50.31,48.55\left(6 \alpha-, 6 \beta-\mathrm{OCH}_{3}\right), 46.76,46.42(2 \mathrm{C}-$ 16), 46.42, 45.15 (2 C-13), 41.21, 40.95 (2 C-15), 29.21, 28.99 (2 C-10); MS: m/z 612. Anal. Calcd. for $\mathrm{C}_{36} \mathrm{H}_{40} \mathrm{~N}_{2} \mathrm{O}_{7}$ (612.72): C, 70.57; H, 6.58; N, 4.57. Found: C, 70.31; H, 6.62; N, 4.65.

(9S)-17-Benzyl-4,5-epoxy-3-methoxy-9-(oxazolidin-2'-on-3'-yl)hasubanan-6-one dimethyl acetal (8a). A mixture of $3 \mathbf{d}(10.3 \mathrm{~g}, 16.8 \mathrm{mmol}), \mathrm{CH}_{2} \mathrm{Cl}_{2}(120 \mathrm{~mL}), p$-toluenesulfonyl chloride (3.2 g, $17 \mathrm{mmol}$, freshly purified), 4-dimethylaminopyridine $(2.1 \mathrm{~g}, 17.2 \mathrm{mmol})$ and triethylamine $\left(5 \mathrm{~mL}, 3.6 \mathrm{~g}, 36 \mathrm{mmol}\right.$ ) was stirred under Ar at $40{ }^{\circ} \mathrm{C}$ (bath temperature) for $10 \mathrm{~h}$. After evaporation of the solvent the residue was dissolved in ethyl acetate, the organic extract was washed with a saturated aqueous $\mathrm{NaHCO}_{3}$ solution, then with brine, dried over $\mathrm{Na}_{2} \mathrm{SO}_{4}$, and 
concentrated under reduced pressure. After purification of the residue by flash chromatography on silica gel (petrol ether/ethyl acetate/triethylamine 6:4:1) colourless crystals 8a were isolated (7.95 g, 94\%); mp 188-190 ${ }^{\circ} \mathrm{C}(\mathrm{MeOH}) ;{ }^{1} \mathrm{H} \mathrm{NMR}\left(\mathrm{CDCl}_{3}\right): \delta 7.39-7.26\left(\mathrm{~m}, 5 \mathrm{H}, \mathrm{C}_{6} \mathrm{H}_{5}\right), 6.75(\mathrm{~d}$, $\left.J_{1,2}=8.2 \mathrm{~Hz}, 1 \mathrm{H}, \mathrm{H}-1\right), 6.71$ (d, $\left.J_{1,2}=8.2 \mathrm{~Hz}, \mathrm{H}, \mathrm{H}-1\right), 6.20$ (d, J7,8 = $\left.10.8 \mathrm{~Hz}, 1 \mathrm{H}, \mathrm{H}-7\right), 6.02$ (d, $\left.J_{7,8}=10.8 \mathrm{~Hz}, 1 \mathrm{H}, \mathrm{H}-8\right), 4.67\left(\mathrm{~d}, J_{\mathrm{gem}}=12 \mathrm{~Hz}, 1 \mathrm{H}\right.$, benzyl-HA), $4.66(\mathrm{~s}, 1 \mathrm{H}, \mathrm{H}-5), 4.57(\mathrm{~m}, 1 \mathrm{H}$,

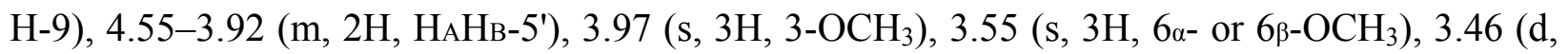
$J_{\text {gem }}=12 \mathrm{~Hz}, 1 \mathrm{H}$, benzyl-HB), 3.49, $2.82\left(\mathrm{~m}, \mathrm{~m}, 1 \mathrm{H}, 1 \mathrm{H}, \mathrm{H}_{\mathrm{AHB}}-10\right), 3.09(\mathrm{~s}, 3 \mathrm{H}, 6 \alpha-$ or $6 \beta-$ $\left.\mathrm{OCH}_{3}\right)$, 2.93, 2.65 (m, m, 1H, 1H, НАНв-4'), 2.77, 2.30 (m, m, 1H, 1H, НАНв-16), 2.09, 1.63 (m, $\left.\mathrm{m}, 1 \mathrm{H}, 1 \mathrm{H}, \mathrm{H}_{\mathrm{AH}}-15\right) .{ }^{13} \mathrm{C} \mathrm{NMR}\left(\mathrm{CDCl}_{3}\right): \delta 159.11$ (C-2'), 146.08 (C-4), 142.09 (C-3), 140.07 (CPh-1), 132.44 (C-12), 130.84, 130.60 (C-7, C-8), 128.11 (CPh-2,3,5,6), 126.77 (CPh-4), 124.39 (C-11), 119.51 (C-1), 115.09 (C-2), 94.47 (C-6), 94.23 (C-5), 67.95 (C-14), 62.18 (C-5'), 57.23 $\left(3-\mathrm{OCH}_{3}\right), 57.13(\mathrm{C}-9), 52.70$ (benzyl- $\left.\mathrm{CH}_{2}\right), 51.75$ (C-13), 50.36, $48.61\left(6 \alpha-, 6 \beta-\mathrm{OCH}_{3}\right), 47.05$ (C-16), 42.14 (C-4'), 41.78 (C-15), 27.90 (C-10); IR (KBr): 1747.8, 1722.3 cm-1; MS: m/z 504. Anal Calcd for $\mathrm{C}_{29} \mathrm{H}_{32} \mathrm{~N}_{2} \mathrm{O}_{6}$ (504.54): C, 69.03; H, 6.39; N, 5.55. Found C, 68.83; H, 6.49; N, 5.38.

\section{$\mathrm{X}$-ray analysis of compound 8a}

With methylene chloride as solvent inclusion $\left(\mathrm{C}_{29} \mathrm{H}_{32} \mathrm{~N}_{2} \mathrm{O}_{6}+\mathrm{CH}_{2} \mathrm{Cl}_{2}, \mathrm{M}=504.54+84.93\right)$. The compound crystallized in monoclinic space group $P 21$ with unit cell parameters $\mathrm{a}=9.440(2), \mathrm{b}=$ 10.059(2), $c=14.889(3) \AA, \beta=95.51(3)^{\circ}, V=1407.3(5) \AA 3, Z=2, D_{c}=1.391 \mathrm{~g} / \mathrm{cm}-3, \mu=0.278$ mm-1. Mo-K $\alpha$ radiation $(\lambda=0.71073 \AA$ ), 5748 reflections measured, 5738 unique, 5549 with $\mathrm{F}=$ $2 \sigma(\mathrm{F})$ gave $\mathrm{R} 1=0.029 / \mathrm{wR}_{2}($ all data $)=0.073$ in a full matrix least squares refinement with 498 parameters. Goodness-of-fit on $\mathrm{F}_{2}=1.073$, absolute structure parameter FLACK $=0.02(4)$. The crystal structure data of 8a have been deposited at the Cambridge Crystallographic Data Centre, 12 Union Road, Cambridge CB2 1EZ, UK; deposition number 155910.

(9S)-4,5-Epoxy-3-methoxy-9-(oxazolidin-2'-on-3'-yl)hasubanan-6-one dimethyl acetal (8b). A solution of 8a (1.45 g, $2.9 \mathrm{mmol})$ and $\mathrm{Pd} / \mathrm{C}$-hydrogenation catalyst (1.5 g, 5\% Pd; Sigma) in acetic acid $(50 \mathrm{~mL})$ was vigorously stirred under hydrogen gas at 1 bar at room temperature for 1h. After filtration through celite on a glass suction sinter funnel the filtrate was concentrated to afford a pale oil of fair purity $(1.08 \mathrm{~g}, 91 \%)$, which crystallized from ethyl acetate; mp 208$211^{\circ} \mathrm{C}$; IR (KBr): $1751.8 \mathrm{~cm}^{-1} ;{ }^{1} \mathrm{H} \mathrm{NMR}\left(\mathrm{CDCl}_{3}\right): \delta 6.70\left(\mathrm{~d}, J_{1,2}=8.1 \mathrm{~Hz}, 1 \mathrm{H}, \mathrm{H}-1\right), 6.66\left(\mathrm{~d}, J_{1,2}\right.$ $=8.1 \mathrm{~Hz}, 1 \mathrm{H}, \mathrm{H}-2), 5.87\left(\mathrm{~d}, J_{7,8}=10.5 \mathrm{~Hz}, 1 \mathrm{H}, \mathrm{H}-8\right), 5.79\left(\mathrm{~d}, J_{7,8}=10.5 \mathrm{~Hz}, 1 \mathrm{H}, \mathrm{H}-7\right), 4.60$ (s, $1 \mathrm{H}, \mathrm{H}-5), 4.34$ (dd, $\left.J_{9,10}=3.0 \mathrm{~Hz}, J_{9,10}=2.3 \mathrm{~Hz}, 1 \mathrm{H}, \mathrm{H}-9\right), 4.06-3.85$ (m, 2H, НАНв-5'), 3.90 (s, $\left.3 \mathrm{H}, 3-\mathrm{OCH}_{3}\right), 3.48\left(\mathrm{~s}, 3 \mathrm{H}, 6 \alpha-\right.$ or $\left.6 \beta-\mathrm{OCH}_{3}\right), 3.33\left(\mathrm{dd}, J_{\mathrm{gem}}=15.9 \mathrm{~Hz}, J_{9,10}=3.0 \mathrm{~Hz}, 1 \mathrm{H}, \mathrm{H}_{\mathrm{A}}-10\right)$, $3.03\left(\mathrm{~s}, 3 \mathrm{H}, 6 \alpha-\right.$ or $\left.6 \beta-\mathrm{OCH}_{3}\right), 2.97-2.91\left(\mathrm{~m}, 1 \mathrm{H}, \mathrm{H}_{\mathrm{A}}-16\right), 2.88-2.80\left(\mathrm{~m}, 1 \mathrm{H}, \mathrm{H}_{\mathrm{A}}-4 \mathrm{\prime}\right), 2.81-2.75$ (m, 1H, Нв-16), 2.73-2.66 (m, 1Н, Нв-10), 2.58-2.51 (m, 1Н, Нв-4'), 2.17-2.11 (dd, Jgem = 12.3 $\left.\mathrm{Hz}, 1 \mathrm{H}, \mathrm{H}_{\mathrm{A}}-15\right), 1.72-1.58$ (m, 1H, Нв-15). ${ }^{13} \mathrm{C} \mathrm{NMR}\left(\mathrm{CDCl}_{3}\right): \delta 158.95$ (C-2'), $146.11(\mathrm{C}-4)$, 141.95 (C-3), 135.03 (C-8), 131.95 (C-12), 127.93 (C-7), 124.25 (C-11), 119.55 (C-1), 114.95 (C-2), 94.80 (C-6), 94.11 (C-5), 66.12 (C-14), 62.03 (C-5'), 59.72 (C-9), 57.07, 50.20, 48.50 (3- 
$\mathrm{OCH}_{3}, 6 \alpha-$ and $\left.6 \beta-\mathrm{OCH}_{3}\right), 50.10$ (C-13), 43.98 (C-15), $41.91,41.87$ (C-16, C-4'), 27.19 (C-10); MS: $m / z$ 414.46. Anal. Calcd. for $\mathrm{C}_{22} \mathrm{H}_{26} \mathrm{~N}_{2} \mathrm{O}_{6}$ (414.46): C, 63.76; H, 6.32; N, 6.76. Found: C, $63.64 ; \mathrm{H}, 6.39 ; \mathrm{N}, 6.70$.

(9S)-4,5-Epoxy-3-methoxy-17-methyl-9-(oxazolidin-2'-on-3'-yl)hasubanan-6-one dimethyl acetal (8c). MeI (1 mL, $2.28 \mathrm{~g}, 16 \mathrm{mmol})$ was added to a solution of $\mathbf{8 b}(410 \mathrm{mg}, 1 \mathrm{mmol})$ in $\mathrm{CH}_{2} \mathrm{Cl}_{2}(8 \mathrm{~mL})$, and the mixture was stirred under $\mathrm{Ar}$ at room temperature for $24 \mathrm{~h}$. After concentrating the solution, the residue was dissolved in ethyl acetate. The solution was placed in a separatory funnel and washed with $2 \mathrm{M} \mathrm{NaOH}$ and subsequently with brine. The organic layer was separated and dried over $\mathrm{Na}_{2} \mathrm{SO}_{4}$. After evaporation of the solvent, the residue was subjected to flash chromatography on silica gel (petrol ether/ethyl acetate/triethylamine $5: 2: 1, \mathrm{R}_{f}=0.35$ ) affording 8c (240 mg, 57\%), which crystallized from methanol; mp 195-197 ${ }^{\circ} \mathrm{C}$; IR $(\mathrm{KBr})$ : $1745.6 \mathrm{~cm}^{-1} ;{ }^{1} \mathrm{H} \mathrm{NMR}\left(\mathrm{CDCl}_{3}\right): \delta 6.68\left(\mathrm{~d}, J_{1,2}=8.2 \mathrm{~Hz}, 1 \mathrm{H}, \mathrm{H}-2\right), 6.63\left(\mathrm{~d}, J_{1,2}=8.2 \mathrm{~Hz}, 1 \mathrm{H}, \mathrm{H}-\right.$ 1), $5.97\left(\mathrm{~d}, J_{7,8}=11.1 \mathrm{~Hz}, 1 \mathrm{H}, \mathrm{H}-8\right), 5.91\left(\mathrm{~d}, J_{7,8}=11.1 \mathrm{~Hz}, 1 \mathrm{H}, \mathrm{H}-7\right), 4.57$ (s, 1H, H-5), 4.35

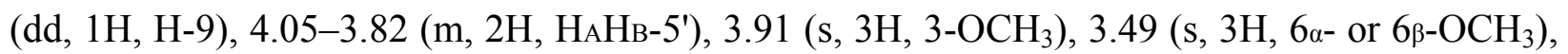
$3.25\left(\mathrm{dd}, J_{\text {gem }}=15.6 \mathrm{~Hz}, J_{9,10}=4.5 \mathrm{~Hz}, 1 \mathrm{H}, \mathrm{HA}-10\right), 3.01\left(\mathrm{~s}, 3 \mathrm{H}, 6 \alpha-\right.$ or $\left.6 \beta-\mathrm{OCH}_{3}\right), 2.87-2.77(\mathrm{~m}$, 2H, HA-4', HA-16), 2.66 (dd, J9,10=2.4 Hz, 1H, Нв-10), $2.58\left(\mathrm{~s}, 3 \mathrm{H}, \mathrm{NCH}_{3}\right), 2.57-2.50$ (m, 1H, Нв-4'), 2.42-2.33 (m, 1Н, Нв-16), 2.09-2.04 (m, 1H, НА-15), 1.67-1.57 (m, 1H, Нв-15). ${ }^{13} \mathrm{C}$ NMR ( $\left.\mathrm{CDCl}_{3}\right): \delta 158.99$ (C-2'), 146.08 (C-4), 142.03 (C-3), 132.35 (C-12), 130.28 (C-7, C-8), 124.53 (C-11), 119.39 (C-1), 115.08 (C-2), 94.31 (C-6), 94.15 (C-5), 67.39 (C-14), 62.11 (C-5'), $57.22\left(3-\mathrm{OCH}_{3}\right), 56.76(\mathrm{C}-9), 51.59(\mathrm{C}-13), 50.82(\mathrm{C}-16), 50.28\left(6 \alpha-\right.$ or $\left.6 \beta-\mathrm{OCH}_{3}\right), 48.51$ (6a- or $\left.6 \beta-\mathrm{OCH}_{3}\right), 42.03\left(\mathrm{C}-4{ }^{\prime}\right), 41.47(\mathrm{C}-15), 35.27\left(\mathrm{NCH}_{3}\right), 27.82$ (C-10). MS: m/z 428. Anal. Calcd. for $\mathrm{C}_{23} \mathrm{H}_{28} \mathrm{~N}_{2} \mathrm{O}_{6}$ (428.48): C, 64.47; H, 6.59; N, 6.54. Found: C, 64.34; H, 6.63; N, 6.41.

(9S) 17-Allyl-4,5-epoxy-3-methoxy-9-(oxazolidin-2'-on-3'-yl)hasubanan-6-one dimethyl acetal (8d). To a solution of $\mathbf{8 b}(320 \mathrm{mg}, 0.8 \mathrm{mmol})$ in $\mathrm{CH}_{2} \mathrm{Cl}_{2}(10 \mathrm{~mL})$ was added 3bromopropene $(0.75 \mathrm{~mL}, 1.05 \mathrm{~g}, 8.7 \mathrm{mmol})$, and the mixture was stirred under $\mathrm{Ar}$ at room temperature for $48 \mathrm{~h}$. After concentrating, the mixture was taken up in ethyl acetate, placed in a separatory funnel, and sodium carbonate ( $2 \mathrm{~mL}, 2 \mathrm{M}$ aqueous solution) was added. Washing the organic layer with brine, drying over $\mathrm{Na}_{2} \mathrm{SO}_{4}$ and evaporation of the solvent furnished a crude oil (240 mg), which was purified by flash chromatography on silica gel (petrol ether/ethyl acetate/triethylamine $\left.3: 2: 1, \mathrm{R}_{f} 0.54\right)$ to yield crystalline 8d $(160 \mathrm{mg}, 46 \%) ; \mathrm{mp} 158-160{ }^{\circ} \mathrm{C}$ (ethyl acetate); IR (KBr): $1736.3 \mathrm{~cm}^{-1} ;{ }^{1} \mathrm{H} \mathrm{NMR}\left(\mathrm{CDCl}_{3}\right): \delta 6.00\left(\mathrm{~d}, J_{1,2}=8.1 \mathrm{~Hz}, 1 \mathrm{H}, \mathrm{H}-2\right), 5.89$ $\left(\mathrm{d}, J_{1,2}=8.1 \mathrm{~Hz}, 1 \mathrm{H}, \mathrm{H}-1\right), 6.01\left(\mathrm{~d}, J_{7,8}=11.1 \mathrm{~Hz}, 1 \mathrm{H}, \mathrm{H}-8\right), 5.89$ (d, $\left.J_{7,8}=11.1 \mathrm{~Hz}, 1 \mathrm{H}, \mathrm{H}-7\right)$, 5.90-5.77 (m, 1H, CH=), $5.18\left(\mathrm{~d}, J_{\mathrm{vic}}=16.8 \mathrm{~Hz}, 1 \mathrm{H},=\mathrm{CHA}\right), 5.06\left(\mathrm{~d}, J_{\mathrm{vic}}=9.9 \mathrm{~Hz}, 1 \mathrm{H},=\mathrm{CHв}\right)$, 4.57 (s, 1H, H-5), 4.33 (dd, 1H, H-9), 4.05-3.82 (m, 3H, НАНв-5', NCHA), 3.90 (s, 3H, 3-OCH $)$, $3.48\left(\mathrm{~s}, 3 \mathrm{H}, 6_{\alpha}-\right.$ or $\left.6 \beta-\mathrm{OCH}_{3}\right), 3.15\left(\mathrm{dd}, 1 \mathrm{H}, \mathrm{H}_{\mathrm{A}}-10\right), 3.01\left(\mathrm{~s}, 3 \mathrm{H}, 6 \alpha-\right.$ or $\left.6 \beta-\mathrm{OCH}_{3}\right), 2.99-2.70(\mathrm{~m}$, $3 \mathrm{H}, \mathrm{H}_{\mathrm{A}}-4$ ', HA-16, NCHв), 2.65 (dd, 1H, Hв-10), 2.60-2.45 (m, 1H, Hв-4'), 2.25-2.18 (m, 1H, Нв-16), 2.08-2.01 (m, 1H, HA-15), 1.64-1.53 (m,1H, Нв-15); ${ }^{13} \mathrm{C}$ NMR $\left(\mathrm{CDCl}_{3}\right): \delta 159.02(\mathrm{C}-$ 2'), 146.01 (C-4), 141.99 (C-3), 137.05 (=CH), 132.38 (C-12), 130.64, 130.18 (C-7, C-8), 124.44 (C-11), 119.38 (C-1), $115.93\left(\mathrm{CH}_{2}\right), 115.05$ (C-2), 94.39 (C-6), 94.12 (C-5), 67.71 (C-14), 62.11 $\left(\mathrm{C}-5{ }^{\prime}\right), 57.18\left(3-\mathrm{OCH}_{3}\right), 57.03(\mathrm{C}-9), 51.70(\mathrm{C}-13), 51.41\left(\mathrm{NCH}_{2}\right), 50.27\left(6 \alpha-\right.$ or $\left.6 \beta-\mathrm{OCH}_{3}\right), 48.51$ 
(6 $\alpha$ - or $\left.6 \beta-\mathrm{OCH}_{3}\right), 47.15$ (C-16), $42.04(\mathrm{C}-4$ ', 41.57 (C-15), 27.74 (C-10). MS: m/z 453. Anal. Calcd. for $\mathrm{C}_{25} \mathrm{H}_{30} \mathrm{~N}_{2} \mathrm{O}_{6}$ (454.52): C, 66.06; H, 6.65; N, 6.16. Found: C, 65.98; H, 6.77; N, 6.21.

$\mathbf{X}$-ray analysis of $8 \mathbf{d}\left(\mathrm{C}_{25} \mathrm{H}_{30} \mathrm{~N}_{2} \mathrm{O}_{6}, \mathrm{M}=454.52\right)$ : The compound crystallized in orthorhombic space group $P 212121$ with unit cell parameters $\mathrm{a}=9.4720(1), \mathrm{b}=11.8370(2), \mathrm{c}=19.8010(3) \AA, \alpha$ $=\beta=\gamma=90^{\circ}, \mathrm{V}=2220.09(6) \AA \AA 3, \mathrm{Z}=4, \mathrm{D}_{\mathrm{c}}=1.360 \mathrm{~g} \mathrm{~cm}-3, \mu=0.090 \mathrm{~mm}-1$. Mo-K $\alpha$ radiation $(\lambda$ $=0.71073 \AA), 6736$ reflections measured, 6711 unique, 6098 with $\mathrm{F}=2 \sigma(\mathrm{F})$ gave $\mathrm{R} 1=0.036$ / $\mathrm{wR}_{2}$ (all data) $=0.0827$ in a full matrix least squares refinement with 419 parameters. Goodnessof-fit on $\mathrm{F}_{2}=1.027$, absolute structure parameter FLACK $=0.0(6)$. The crystal structure of 8d has been deposited at the Cambridge Crystallographic Data Centre, 12 Union Road, Cambridge CB2 1EZ, UK; deposition number 155909.

(9S)-17-Benzyl-4,5-epoxy-3-methoxy-9-(oxazolidin-2'-on-3'-yl)hasubanan-6-one (8e). A solution of 8a $(1.22 \mathrm{~g}, 2.4 \mathrm{mmol})$ in $\mathrm{EtOH}(10 \mathrm{~mL})$ and $1 \mathrm{~N} \mathrm{HCl}(3 \mathrm{~mL})$ was heated for $5 \mathrm{~min}$. After cooling the mixture was made alkaline with $2 \mathrm{M} \mathrm{NaOH}(4 \mathrm{~mL})$ and extracted with ethyl acetate. The extract was washed with water and dried over $\mathrm{Na}_{2} \mathrm{SO}_{4}$ to yield colourless crystals $8 \mathrm{e}$ (1.07 g, 97\%); mp 134-136 ${ }^{\circ} \mathrm{C}(\mathrm{MeOH})$; IR (KBr): 1724.1, $1711.2 \mathrm{~cm}^{-1} ;{ }^{1} \mathrm{H} \mathrm{NMR}\left(\mathrm{CDCl}_{3}\right): \delta$ 7.36-7.27 (m, 5H, $\left.\mathrm{C}_{6} \mathrm{H}_{5}\right), 7.04\left(\mathrm{~d}, J_{7,8}=10.8 \mathrm{~Hz}, 1 \mathrm{H}, \mathrm{H}-8\right), 6.77$ (d, J1,2 = 8.1 Hz, 1H, H-1), 6.74 $\left(\mathrm{d}, J_{1,2}=8.1 \mathrm{~Hz}, 1 \mathrm{H}, \mathrm{H}-2\right), 6.28$ (d, $\left.J_{1,2}=8.1 \mathrm{~Hz}, 1 \mathrm{H}, \mathrm{H}-7\right), 4.84$ (s, 1H, H-5), 4.68 (d, $J_{\text {gem }}=13.2$ Hz, 1H, benzyl-HA), 4.68 (m, 1H, H-9), 3.97-3.77 (m, 2H, НАНв-5'), 3.92 (s, 3H, 3-OCH3), 3.53 $\left(\mathrm{d}, J_{\text {gem }}=13.2 \mathrm{~Hz}, 1 \mathrm{H}\right.$, benzyl-Нв), 3.50 (m, 1H, НА-10), 2.91 (m, 2H, Нв-10, НА-16), 2.63 (m, 1H, HA-4'), 2.51 (m, 1H, Нв-16), 2.22 (m, 1H, HA-15), 1.96 (m, 1H, Нв-4'), 1.77 (m, 1H, Нв-15). ${ }^{13} \mathrm{C}$ NMR $\left(\mathrm{CDCl}_{3}\right): \delta 195.16(\mathrm{C}-6), 159.02$ (C-2'), 148.37 (C-8), $144.80(\mathrm{C}-4), 143.38(\mathrm{C}-3)$, 139.20 (CPh-1), 130.02 (C-12), 129.59 (C-7), 128,40, $128.10(\mathrm{CPh}-2,3,5,6), 127.19(\mathrm{CPh}-4)$, 124.12 (C-11), 120.85 (C-1), 114.36 (C-2), 89.71 (C-5), 67.82 (C-14), 62.03 (C-5'), 57.11 (C-9), $56.58\left(3-\mathrm{OCH}_{3}\right), 54.07$ (C-13), 52.85 (benzyl-CH$), 48.13$ (C-16), 41.76 (C-4'), 28.47 (C-10); MS: $m / z$ 458. Anal. Calcd. for $\mathrm{C}_{27} \mathrm{H}_{26} \mathrm{~N}_{2} \mathrm{O}_{5}$ (458.51): C, 70.73; H, 5.72; N, 6.11. Found: C, $70.51 ; \mathrm{H}, 5.81 ; \mathrm{N}, 5.98$.

(9S)-4,5-Epoxy-3-methoxy-9-(oxazolidin-2'-on-3'-yl)hasubanan-6-one (8f). A solution of 8b $(550 \mathrm{mg}, 1.3 \mathrm{mmol})$ in $1 \mathrm{~N} \mathrm{HCl}(4 \mathrm{~mL})$ was heated for $5 \mathrm{~min}$. After cooling, the mixture was made alkaline with $2 \mathrm{M} \mathrm{NaOH}(3 \mathrm{~mL})$ and extracted with ethyl acetate. The organic extract was washed with brine, dried over $\mathrm{Na}_{2} \mathrm{SO}_{4}$, and after evaporation of the solvent pale yellow crystals 8f (470 mg, 96\%) were obtained; $\mathrm{mp} 213-215{ }^{\circ} \mathrm{C}(\mathrm{MeOH})$; IR (KBr): 3440.3, 3296.9, 1745.1, $1704.4 \mathrm{~cm}^{-1} ;{ }^{1} \mathrm{H}$ NMR $\left(\mathrm{CDCl}_{3}\right): \delta 6.73(\mathrm{~s}, 2 \mathrm{H}, \mathrm{H}-1, \mathrm{H}-2), 6.68(\mathrm{~d}, J 7,8=10.8 \mathrm{~Hz}, 1 \mathrm{H}, \mathrm{H}-8), 6.09$ $\left(\mathrm{d}, J_{7,8}=10.8 \mathrm{~Hz}, 1 \mathrm{H}, \mathrm{H}-7\right), 4.81(\mathrm{~s}, 1 \mathrm{H}, \mathrm{H}-5), 4.46$ (m, 1H, H-9), 3.90 (s, 3H, 3-OCH3), 3.93$3.70\left(\mathrm{~m}, 2 \mathrm{H}, \mathrm{H}_{\mathrm{A}} \mathrm{HB}_{-} 5^{\prime}\right), 3.34\left(\mathrm{dd}, J_{\mathrm{gem}}=15.9 \mathrm{~Hz}, J_{9,10}=4.2 \mathrm{~Hz}, 1 \mathrm{H}, \mathrm{H}_{\mathrm{A}}-10\right), 3.14-2.95$ (m, 2H, НАНв-16), 2.72 (dd, J9,10 = 2.4 Hz, 1H, Нв-10), 2.58-2.42 (m, 1H, HA-4'), 2.29 (dd, Jgem $=12.6$ $\left.\mathrm{Hz}, J_{15 \mathrm{~A}, 16}=3.9 \mathrm{~Hz}, 1 \mathrm{H}, \mathrm{HA}-15\right), 1.96-1.87$ (m, 1H, Нв-4'), 1.86-1.77 (m, 1H, Нв-15). ${ }^{13} \mathrm{C}$ NMR $\left(\mathrm{CDCl}_{3}\right): \delta 195.23(\mathrm{C}-6), 158.88\left(\mathrm{C}-2^{\prime}\right), 150.32$ (C-8), 144.99 (C-4), 143.29 (C-3), $129.67(\mathrm{C}-$ 12), 126.73 (C-7), 124.10 (C-11), 120.88 (C-1), 114.24 (C-2), 89.40 (C-5), 65.73 (C-14), 61.95 (C-5'), 59.67 (C-9), $56.50\left(3-\mathrm{OCH}_{3}\right), 52.42$ (C-13), 43.17 (C-15, C-16), 41.54 (C-4'), 27.95 (C10); MS: m/z 368. Anal. Calcd. for $\mathrm{C}_{20} \mathrm{H}_{20} \mathrm{~N}_{2} \mathrm{O}_{5}$ (368.39): C, 65.21; H, 5.47; N, 7.60. Found: $\mathrm{C}$, 
$65.01 ; \mathrm{H}, 5.64 ; \mathrm{N}, 7.41$.

(9S)-4,5-Epoxy-3-methoxy-17-methyl-9-(oxazolidin-2'-on-3'-yl)hasubanan-6-one (8g). A solution of $\mathbf{8 c}(100 \mathrm{mg}, 0.23 \mathrm{mmol})$ in $1 \mathrm{~N} \mathrm{HCl}(3 \mathrm{~mL})$ was heated for $5 \mathrm{~min}$. After cooling the mixture was made alkaline with $2 \mathrm{M} \mathrm{NaOH}(3 \mathrm{~mL})$ and extracted with ethyl acetate. The extract was washed with brine and dried over $\mathrm{Na}_{2} \mathrm{SO}_{4}$. After evaporation of the solvent colourless crystals $8 \mathrm{~g}$ (80 mg, 90\%) were obtained; mp 209-211 ${ }^{\circ} \mathrm{C}$ (ethyl acetate); IR (KBr): 1724.2, $1706.6 \mathrm{~cm}^{-1} ;{ }^{1} \mathrm{H}$ NMR $\left(\mathrm{CDCl}_{3}\right): \delta 6.93\left(\mathrm{~d}, J_{7,8}=10.8 \mathrm{~Hz}, 1 \mathrm{H}, \mathrm{H}-8\right), 6.71$ (s, 2H, H-1, H-2), 6.20 $\left(\mathrm{d}, J_{7,8}=10.8 \mathrm{~Hz}, 1 \mathrm{H}, \mathrm{H}-7\right), 4.78$ (s, 1H, H-5), 4.48 (dd, 1H, H-9), 3.89 (s, 3H, 3-OCH $), 3.92-$ $3.70\left(\mathrm{~m}, 2 \mathrm{H}, \mathrm{HAHB}_{\mathrm{A}} 5^{\prime}\right), 3.26\left(\mathrm{dd}, J_{\mathrm{gem}}=15.6 \mathrm{~Hz}, J_{9,10}=4.5 \mathrm{~Hz}, 1 \mathrm{H}, \mathrm{HA}_{\mathrm{A}} 10\right), 2.96$ (dd, 1H, HA16), $2.70\left(\mathrm{dd}, J_{\mathrm{gem}}=15.6 \mathrm{~Hz}, J_{9,10}=2.4 \mathrm{~Hz}, 1 \mathrm{H}, \mathrm{HB}-10\right), 2.67\left(\mathrm{~s}, 3 \mathrm{H}, \mathrm{NCH}_{3}\right), 2.64$ (m, 1H, Hв16), 2.57 (m, 1H, HA-4'), 2.21 (m, 1H, HA-15), 1.91 (m, 1H, HB-4'), 1.79 (m, 1H, HB-15); ${ }^{13} \mathrm{C}$ NMR $\left(\mathrm{CDCl}_{3}\right): \delta 195.04$ (C-6), 158.86 (C-2'), 148.28 (C-8), 144.71 (C-4), 143.21 (C-3), 129.98 (C-12), 129.36 (C-7), 124.22 (C-11), 120.70 (C-1), 114.21 (C-2), 89.59 (C-5), 67.27 (C-14), 61.89 (C-5'), 56.75 (C-9), 56.49 (3-OCH3), 53.82 (C-13), 51.87 (C-16), 41.62 (C-4'), 40.17 (C15), 35.42 (17- $\mathrm{NCH}_{3}$ ), 28.32 (C-10); MS: m/z 382. Anal. Calcd. for $\mathrm{C}_{21} \mathrm{H}_{22} \mathrm{~N}_{2} \mathrm{O}_{5}$ (382.42): C, 65.96; H, 5.80; N, 7.33. Found: C, 65.69; H, 5.97; N, 7.17.

(9S)-17-Allyl-4,5-epoxy-3-methoxy-9-(oxazolidin-2'-on-3'-yl)hasubanan-6-one (8h). A solution of $\mathbf{8 d}(100 \mathrm{mg}, 0.22 \mathrm{mmol})$ in $1 \mathrm{~N} \mathrm{HCl}(3 \mathrm{~mL})$ was heated for $5 \mathrm{~min}$. After cooling, the mixture was made alkaline with $2 \mathrm{M} \mathrm{NaOH}(3 \mathrm{~mL})$ and extracted with ethyl acetate. The extract was washed with brine, dried over $\mathrm{Na}_{2} \mathrm{SO}_{4}$. After evaporation of the solvent, $8 \mathbf{h}(80 \mathrm{mg}, 89 \%)$ was obtained as solid material; IR (KBr): 1738.4, $1701.2 \mathrm{~cm}^{-1} ;{ }^{1} \mathrm{H} \mathrm{NMR}\left(\mathrm{CDCl}_{3}\right): \delta 6.90(\mathrm{~d}, J 7,8=$ $10.8 \mathrm{~Hz}, 1 \mathrm{H}, \mathrm{H}-8$ ), 6.71 (s, 2H, H-1, H-2), 6.18 (d, J7,8 = 10.8 Hz, 1H, H-7), 6.22-5.76 (m, 1H, $=\mathrm{CH}), 5.24\left(\mathrm{~d}, J_{\mathrm{vic}}=17.1 \mathrm{~Hz}, 1 \mathrm{H},=\mathrm{CHA}\right), 5.12\left(\mathrm{~d}, J_{\mathrm{vic}}=10.2 \mathrm{~Hz} ; 1 \mathrm{H},=\mathrm{CHB}\right), 4.78(\mathrm{~s}, 1 \mathrm{H}, \mathrm{H}-5)$, 4.48 (m, 1H, H-9), 3.98 (m, 1H, NCHA), 3.95-3.68 (m, 2H, НАНв-5'), 3.89 (s, 3H, 3-OCH3), $3.31\left(\mathrm{dd}, J_{\text {gem }}=15.7 \mathrm{~Hz}, \mathrm{~J}_{9,10}=4.5 \mathrm{~Hz}, 1 \mathrm{H}, \mathrm{HA}_{\mathrm{A}} 10\right), 3.11-3.04$ (m, 2H, HA-16, NCHв), 2.70 (dd, $\left.J_{\text {gem }}=15.7 \mathrm{~Hz}, J_{9,10}=2.5 \mathrm{~Hz}, 1 \mathrm{H}, \mathrm{HB}_{-10}\right), 2.59-2.38$ (m, 2H, НА-4', Нв-16), 2.21 (m, 1H, НА15), 1.90 (m, 1H, Hв-4'), 1.76 (m, 1H, Нв-15); ${ }^{13} \mathrm{C} \mathrm{NMR}\left(\mathrm{CDCl}_{3}\right): \delta 195.09$ (C-6), $158.90\left(\mathrm{C}-2^{\prime}\right)$, 148.27 (C-8), 144.68 (C-4), 143.21 (C-3), 136.25 (=CH), 129.93 (C-12), 129.14 (C-7), 124.15 (C-11), $120.71(\mathrm{C}-1), 116.79\left(=\mathrm{CH}_{2}\right), 114.23$ (C-2), 89.54 (5), 67.50 (C-14), 61.93 (C-5'), 56.99 (C-9), $56.49\left(3-\mathrm{OCH}_{3}\right), 54.00(\mathrm{C}-13), 51.49\left(\mathrm{NCH}_{2}\right), 48.22(\mathrm{C}-16), 41.63(\mathrm{C}-4), 40.23(\mathrm{C}-15)$, 28.27 (C-10); MS: m/z 408. Anal. Calcd. for $\mathrm{C}_{23} \mathrm{H}_{24} \mathrm{~N}_{2} \mathrm{O}_{5}$ (408.45): C, 67.63; H, 5.92; N, 6.86. Found: C, 67.54; H, 6.21; N, 6.92.

(9S) 17-Benzyloxycarbonyl-4,5-epoxy-3-methoxy-9-(oxazolidin-2'-on-3'-yl)hasuban-an-6one (8i). To a solution of $\mathbf{8 b}(250 \mathrm{mg}, 0.6 \mathrm{mmol})$ in acetone $(20 \mathrm{~mL})$ was added $\mathrm{K}_{2} \mathrm{CO}_{3}(100 \mathrm{mg})$ and benzyl chloroformate $(0.1 \mathrm{~mL}, 120 \mathrm{mg}, 0.7 \mathrm{mmol})$. The mixture was stirred under Ar at room temperature for $10 \mathrm{~h}$. After concentration under reduced pressure the residue was extracted with ethyl acetate. The extract was washed with brine, dried over $\mathrm{Na}_{2} \mathrm{SO}_{4}$, and after removal of the solvent an orange oil $\mathbf{8 i}(330 \mathrm{mg}, 100 \%)$ was isolated which was purified by flash chromatography on silica gel (petrol ether/ethyl acetate/triethylamine $3: 2: 1, \mathrm{R}_{f} 0.22$ ); IR (KBr): 1754.4, $1698.5 \mathrm{~cm}^{-1} ;{ }^{1} \mathrm{H} \mathrm{NMR}\left(\mathrm{CDCl}_{3}\right) \delta$ : 7.44-7.23 (m, 5H, $\left.\mathrm{C}_{6} \mathrm{H}_{5}\right), 6.74\left(\mathrm{~d}, J_{1,2}=8.1 \mathrm{~Hz}, 1 \mathrm{H}, \mathrm{H}-\right.$ 
2), $6.66\left(\mathrm{~d}, J_{1,2}=8.1 \mathrm{~Hz}, 1 \mathrm{H}, \mathrm{H}-1\right), 6.43\left(\mathrm{~d}, J_{7,8}=10.4 \mathrm{~Hz}, 1 \mathrm{H}, \mathrm{H}-8\right), 5.79$ (d, 1H, H-7), 5.31 (m, 1H, H-9), 5.21 (broad s, 2H, benzyl-НАНв), 4.71 (s, 1H, H-5), 4.10-3.91 (m, 2H, НАНв-5'), 3.92 $\left(\mathrm{s}, 3 \mathrm{H}, 3-\mathrm{OCH}_{3}\right), 3.86-3.39$ (m, 2H, НАНB-16), 3.29, $3.23\left(2 \mathrm{~s}, 3 \mathrm{H}, 3 \mathrm{H}, 6 \alpha-\right.$ and $\left.6 \beta-\mathrm{OCH}_{3}\right), 3.48$, 2.97 (2 m, 1H, 1Н, НАНв-10), 2.95 (m, 2H, НАНв-4'), 2.26-2.03 (m, 2H, НАНв-15); ${ }^{13} \mathrm{C}$ NMR $\left(\mathrm{CDCl}_{3}\right): \delta 158.23\left(\mathrm{C}-2^{\prime}\right), 154.29(\mathrm{NCO}), 146.94(\mathrm{C}-4), 142.65$ (C-3), $136.89(\mathrm{Cph}-1), 129.93(\mathrm{C}-$ 12), 129.86, 129.62 (C-7, C-8), 128.32 (CPh-2,3,5,6), 127.85 (CPh-4), 124.66 (C-11), 119.94 (C1), 116.48 (C-2), 94.82 (C-6), 93.03 (C-5), 66.97 (benzyl- $\left.\mathrm{CH}_{2}\right), 65.97$ (C-14), 62.06 (C-5'), 57.35 $\left(3-\mathrm{OCH}_{3}\right), 55.93(\mathrm{C}-9), 54.65(\mathrm{C}-13), 49.39,48.89$ (6 $\alpha-$ and $\left.6 \beta-\mathrm{OCH}_{3}\right), 44.91(\mathrm{C}-16), 42.29(\mathrm{C}-$ 4 ), 38.72 (C-15), 28.84 (C-10); MS: $m / z$ 548, $\mathrm{C}_{30} \mathrm{H}_{32} \mathrm{~N}_{2} \mathrm{O}_{8}$ (548.59).

\section{X-ray structure analysis}

Crystallography. Sample preparation. Crystals suitable for X-ray investigation were prepared by slow evaporation of solutions of the respective compounds. Colourless crystals were grown from ethyl acetate in the case of 8d. Crystallization from $\mathrm{CH}_{2} \mathrm{Cl}_{2}$ gave the clathrate (inclusion compound) $8 \mathbf{a} \cdot \mathrm{CH}_{2} \mathrm{Cl}_{2}(1: 1)$.

Data collection, structure analysis, and refinement. Intensity data for $\mathbf{8 a}$ and $\mathbf{8 d}$ where obtained on a Nonius Kappa instrument with CCD detector at $110 \mathrm{~K}$ (in detail: 8a/8d, collection time per frame 65/50 sec., rotation width 1.5/1.0 deg., detector distance 35/35 mm, total number of frames 570/392). The structures $8 \mathbf{a}$ and $\mathbf{8 d}$ were solved by direct methods, using the program SIR926 and refined with SHELXL93. ${ }^{7}$ The figures were prepared using DIAMOND 2.1a. ${ }^{8}$

\section{References and Notes}

1. (a) Portoghese, P. S. J. Med. Chem. 1992, 35, 1927. (b) Portoghese, P. S. Trends in Pharmacological Sciences 1989, 10, 230.

2. (a) Fleischhacker, W.; Richter, B. Sci. Pharm. 1989, 57, 351. (b) Fleischhacker, W.; Richter, B.; Völlenkle, H. Monatsh.Chem. 1991, 122, 399.

3. Czock, A. Diploma Thesis 1993, University of Vienna.

4. (a) Fleischhacker, W.; Markut, H.; Vieböck, F. Monatsh. Chem. 1972, 133, 1066. (b) Gruber, H. Diploma Thesis 1972, University of Vienna.

5. (a) Fleischhacker, W.; Richter, B. Chem. Ber. 1979, 112, 2539. (b) Fleischhacker, W.; Richter, B. Monatsh. Chem. 1992, 837.

6. SIR92 - A program for crystal structure solution. Altomare, A.; Cascarano, G.; Giacovazzo, C.; Guagliardi, A. J. Appl. Crystallogr. 1993, 26, 343.

7. SHELX97 - Program for Crystal Structure Analysis (Release 97-2). Sheldrick, G. M.; Institut für Anorganische Chemie der Universität, Tammanstrasse 4, D-3400 Göttingen, Germany, 1998.

8. DIAMOND 2.1a - Visual crystal structure information system, Brandenburg, K. 1999. 